\title{
Human herpesvirus 8 (HHV-8) salivary shedding in mothers and children from Uganda: risk factors and clues about transmission
}

\author{
Rita Romano ${ }^{1}$ \\ Silvia Gramolelli ${ }^{1}$ \\ Francesca Tabacchi ${ }^{1}$ \\ Gianluca Russo ${ }^{1}$ \\ Simona Verzaro ${ }^{1}$ \\ Francesco Marinucci² \\ Giacomo Maria Paganotti1 \\ Aurelia Gaeta ${ }^{1}$ \\ Mario Coluzzi ${ }^{1}$
}

1Department of Public Health and Infectious Diseases, "Sapienza", University of Rome, Italy

${ }^{2}$ Institute of Human Virology, University of Maryland

School of Medicine, Baltimore, USA

\section{Corresponding Author:}

Rita Romano

Department of Public Health and Infectious Diseases,

"Sapienza", University of Rome

Piazzale Aldo Moro 5

00185 Rome, Italy

E-mail: rita.romano@uniroma1.it

\begin{abstract}
Background: in Africa, the increase of Kaposi Sarcoma-associated herpesvirus (KSHV/HHV-8) seroprevalence during childhood suggests an horizontal intrafamilial transmission. However, the exact transmission modes are yet unknown, but there is evidence that the virus is intermittently spread through the saliva of seropositive subjects. Furthermore, a significant correlation was found between the geographical distribution of bloodsucking arthropods and incidence rates of Kaposi Sarcoma. In this view, key roles can be played by: i) the inflammatory reaction due to the arthropod bites which create a micro-environment favourable to viral replication, and ii) the use of saliva as firstaid medication in several circumstances. According to that, the viral transmission could occur through the application of mothers' saliva on children's skin to relieve itching and scratching after a bloodsucking arthropod bite. This work investigated the risk factors involved in HHV-8 transmission based on the "promoter arthropod hypothesis". Methods: one hundred and thirty questionnaires were administered to Ugandan children (54 from settled and $\mathbf{7 6}$ from nomad communities) to evaluate the skin inflammatory reaction to the arthropod
\end{abstract}

bites and the frequency of behavioural practices associated with saliva usage. At the same time saliva samples were collected from the children and respective mothers to detect the presence of HHV-8 DNA by Real-Time PCR.

Results: we detected HHV-8 DNA in $23.85 \%$ of mothers and in $11.54 \%$ of children. The use of saliva is significantly more common in settled than in the nomad communities $(p<0.001)$. The statistical analysis showed significant association between: i) skin inflammatory reaction and use of saliva in both groups; ii) environmental factors which increase the presence of bloodsucking arthropods and skin inflammatory reaction.

Discussion: from our data we suppose that HHV-8 transmission could occur through non-sexual routes and that the infected saliva applied at child bite site could represent a favourable condition for the acquiring of the infection.

KEY WORDS: arthropods, HHV-8, Real-Time PCR, saliva, transmission.

\section{Background}

The human herpesvirus 8 (HHV-8) is the causal agent of Kaposi sarcoma (KS) (1) and is also involved in the pathogenesis of two rare lymphoproliferative disorders: Multicentric Castleman disease (MCD) (2) and Primary Effusion Lymphoma (PEL) (3). In sub-Saharan Africa, after the onset of AIDS epidemic, KS became the most common malignancy among adults and the second most prevalent cancer type in childhood $(4,5)$. Thus, $\mathrm{KS}$ emerges as an important problem in sub-Saharan African public health; the investigation of HHV-8 transmission route in these areas represents a key factor in the reduction of $\mathrm{KS}$ incidence.

HHV-8 seroprevalence in sub-Saharan Africa ranges from 40 to $70 \%$ in adults (6-8) while in Mediterranean countries is reported to be $16.5-18.7 \%(8)$. Interestingly, in Amerindian tribes HHV-8 is hyperendemic showing the highest infection rates ever reported (about $80 \%$ in adults) $(9,10)$. In Uganda HHV-8 seroprevalence ranges from $26.6 \%$ in children to $40.1 \%$ in adults showing an age dependent increase during childhood $(7,11,12)$. In several studies is reported that saliva is the major reservoir of HHV-8 $(5,9,12)$. Salivary secretions of seropositive subjects intermittently harbour the virus at greatest concentrations $(13,14)$. Despite in adulthood the transmission is associated both to unprotected sexual activity and drug injection (15), among children a primary role could be played by sali- 
vary transmission (11-13). In particular, the contagion could occur through exposure to infected saliva from mothers or other family members (12), even if little is known about the behavioural practices that expose children to saliva (16). In African countries the use of traditional methods is very common, this practices mainly consist on the application of either saliva or premasticated herbs on child's skin as first-aid medication in several circumstances (16-18).

One hypothesis is that HHV-8 could be transmitted by a seropositive mother to the child through the application of the infected saliva on child's skin to relieve itching and scratching due to a bloodsucking arthropod bite. These arthropods does not inject the virus, prepare instead the cellular and biochemical skin environment promoting the virus infection (19). During the blood meal, an arthropod inoculates antihaemostatic and immunogenic molecules which evoke an immune response at the bite site with release of Th2 cytokines (20). As HHV-8 is avid in these cytokines, viral transmission could be facilitated by a strong inflammatory reaction $(9,21,22)$. Furthermore, several works underlined a significant correlation between the geographical distribution of bloodsucking arthropods and incidence rates of $\mathrm{KS}(21,22)$. According to that, the arthropod, through its biting, increases HHV-8 transmission risk, acquiring the role of "promoter of the infection" (19).

In this study, according to this hypothesis, the risk factors involved in HHV-8 transmission had been investigated: the presence of HHV-8 DNA in saliva of mothers and respective children, the use of maternal saliva to soothe children's itchy bites, the skin inflammatory reaction at children's bite site as well as the presence of environmental factors which could increase the presence of bloodsucking arthropods.

Furthermore, to reveal differences in HHV-8 spreading and saliva-based behavioural practices, we included in the study two groups: settled and nomad communities from Uganda. The former group lived mainly in urban contexts showing sedentary habits, whereas, the latter owned to a nomadic community which was moving throughout Karamoja region to feed livestock.

\section{Methods}

Asintomatic mother and child couples were enrolled consecutively in the different site of study during local information, education and communication (IEC) campaign. The children age was between 3-13 years and only couples with on going breastfeeding were excluded.

\section{Study area and subjects}

One hundred and thirty Ugandan children and respective mothers had been enrolled in the study in 2007 and 2008. The questionnaires were administered at children (3-13 years old) in schools or health care centres. Moreover, saliva samples from the interviewed children and mothers had been collected, spotted on filter paper, air dried and then separately stored in plastic envelopes.

Formal permission and signed informed consent were respectively obtained from the local authorities (school and health care service directors) and from parents or caregivers of the children.

Study participants were recruited in settled (54 children and 54 mothers) and nomad (76 children and 76 mothers) communities, the former from Kampala-Great Valley children centre, Moroto-Kakoliye and Kasimeri primary schools; the latter from Rupa-Kidepo health centre and Namalu health centre in rural Karamoja.

\section{Questionnaires}

Questionnaires were developed by one of the authors (Romano R). The first section included sociodemographic questions about age, gender, lifestyle (i.e. nomad or settled community, access to water facilities and presence of stagnant or running water in the neighbourhood), presence of domestic animals (i.e. hens, cows, sheep, goats, donkeys). The second section included questions about possible promoter arthropods (i.e. perception of insects in the environment, bite frequency, part of the body most bitten). The third section included questions on bitten skin reaction (i.e. itchiness, pain, irritation, swelling, skin mark, lasting of reaction). The last section concerned treatment received (i.e. traditional methods including saliva and premasticated herbs or pharmaceutical products).

\section{DNA extraction}

Each sample had been observed through an UV lamp to detect the exact position of the salivary fluid on filter paper, then the spot (e.g. circle) was excised and placed into $2.0 \mathrm{ml}$ lysis buffer for elution from the filter card by overnight gently rocking at room temperature. Then the paper was removed from the tube and the samples were immediately processed. DNA was extracted by an automatic nucleic acid extractor (Nuclisens EasyMag - Biomerieux). Each specimen samples added with $65 \mu \mathrm{l}$ of magnetic silica, $10 \mu \mathrm{l}$ of internal extraction control (CPE, Nanogen Advanced Diagnostics), represented by purified beta globin sequence, and $55 \mu$ l of wash reagent was loaded in the instrument; DNA was automatically extracted, eluted in $55 \mu \mathrm{l}$ of specific buffer and immediately used in Real Time $\mathrm{PCR}$, an aliquot was stored at $-80^{\circ} \mathrm{C}$.

\section{Real Time PCR}

Extracted DNA was analyzed for the presence of HHV8 by Qualitative TaqMan Real Time PCR using a commercially available kit (Nanogen Advanced Diagnostics, Italy). The assay was performed by a multiplex format targeting both the sequence of HHV8 capsid protein gp27 and beta globin control gene by using 
Human herpesvirus 8 (HHV-8) salivary shedding in mothers and children from Uganda: risk factors and clues about transmission

5' reporter dye 6-carboxyfluorescein (FAM)-labelled probe for viral gene and 5' fluorescein (VIC)-labelled probe for human beta globin respectively. Each PCR reaction was carried out with ABI PRISM 7000 (Applied Biosystem, USA) in 96-well plate, adding $5 \mu \mathrm{l}$ of extracted DNA to $20 \mu \mathrm{l}$ of amplification mixture. In each run HHV8 specific standard constructed with dilutions of plasmids carrying the specific viral gene was added. The standard was used as positive amplification control. The real time PCR thermal profile consisted of a first cycle at $50^{\circ} \mathrm{C}$ for 2 minutes, a second cycle at $95^{\circ} \mathrm{C}$ for 10 minutes, and a third step which included 45 cycles at $95^{\circ} \mathrm{C}$ for 15 seconds and $60^{\circ} \mathrm{C}$ for $1 \mathrm{~min}$. The analysis was performed in two hours for single run and, at the end of the process, computer reported as "positives" the samples that showed HHV-8 DNA presence.

\section{Data analysis}

SPSS, Version 16, was used for statistical analyses. In both communities descriptive statistics were calculated for each question related to the type of treatment received (saliva and premasticated herbs or pharmacological products) and risk factors. Yates-corrected $\mathrm{X}^{2}$ test, calculated odds ratios (OR) with their corresponding 95\% confidence intervals $(\mathrm{Cl})$, were used to investigate factors associated with traditional methods. Furthermore, binary logistic regression analysis (BLR) was used to investigate the impact of different independent risk factors on the dependent dichotomous variable "use of traditional methods". Risk factors included symptoms related to the bite (itchiness, pain, irritation, swelling, skin mark and duration of reaction) and environmental factors (stagnant water and domestic/courtyard animals) which increase the presence of bloodsucking arthropods. These factors represent the most important ones related to the HHV-8 transmission route through promoter arthropods. We report only significant associations $(p<0.05)$. Sample sizes differ slightly between tests because of missing values due to lack of answers.

\section{Results}

The median age of the children was 6.95 years (range, 3-13 years) and $43 \%$ (56/130) were male. Children from settled and nomad communities did not significantly differ in the distribution of age and gender. Data about mother's age were not collected.

Frequencies of questionnaires answers directed to children and related to risk factors involved in HHV-8 transmission are shown in Table 1.

The majority of children $(99.6 \%=129 / 130)$ declared to have been bitten (data not shown) and $35.45 \%$ (39/110) of them receive traditional methods as treatment. Furthermore, among the symptoms of the inflammatory skin reaction, itching is reported to be the most widespread $(61.42 \%)$ and more than a half of children were exposed to both selected environmental factors (Tab. 1).

Table 1 - Frequencies of children's questionnaire answers related possible risk factors involved in HHV-8 transmission.

\begin{tabular}{llll}
\hline Community & Settled $\%(\mathbf{n} / \mathbf{N}) \pm 95 \% \mathbf{C l}$ & Nomadic $\%(\mathbf{n} / \mathbf{N}) \pm 95 \% \mathbf{C l}$ & Total sample $\%(\mathbf{n} / \mathbf{N}) \pm 95 \% \mathbf{C l}$ \\
\hline Traditional methods $\#$ & 51.22 & 26.10 & 35.45 \\
& $(21 / 41) \pm 0.14$ & $(18 / 69) \pm 0.10$ & $(39 / 110) \pm 0.09$ \\
\hline Remedies & & & \\
\hline Pharmaceutical products & 40.74 & 30.3 & 35.42 \\
& $(11 / 27) \pm 0.18$ & $(23 / 69) \pm 0.12$ & $(34 / 96) \pm 0.10$ \\
\hline Irritation & 34.62 & 31.78 & 32.54 \\
& $(18 / 52) \pm 0.14$ & $(23 / 74) \pm 0.10$ & $(23 / 74) \pm 0.08$ \\
\hline Swelling & 88.45 & 47.29 & 48.41 \\
& $(46 / 52) \pm 0.09$ & $(35 / 74) \pm 0.12$ & $(61 / 126) \pm 0.09$ \\
\hline Skin reaction & & & 61.42 \\
\hline Itching & & $(78 / 127) \pm 0.09$ \\
\hline Pain & 92.45 & 39.19 & 36.22 \\
& $(49 / 53) \pm 0.07$ & $(29 / 74) \pm 0.11$ & $(46 / 127) \pm 0.09$ \\
\hline Domestic/courtyard animals & 46.15 & 48.65 & 73.72 \\
& $(24 / 52) \pm 0.14$ & $(36 / 74) \pm 0.12$ & $(92 / 126) \pm 0.03$ \\
\hline Environmental factors & & 91.89 & \\
\hline Stagnant or running & $(10 / 53) \pm 0.11$ & $(68 / 74) \pm 0.01$ & 50.94 \\
water nearby home location & $(21 / 53) \pm 0.13$ & & $(78 / 128) \pm 0.09$ \\
\hline
\end{tabular}

\#: use of saliva and/or premasticated herbs; $\mathrm{Cl}=$ confidence interval.

Statistical comparisons are reported in the main text. 


\section{R. Romano et al.}

In the total sample of children enrolled, the BLR analysis showed that the use of traditional method is directly associated with symptoms of skin reaction such as irritation (OR: 2.96; 95\% Cl: 2.41-153.91; $p=0.04$ ) and swelling (OR: 3.17; 95\% Cl: 2.95-191.18; $p=0.03$ ). Moreover, statistical analysis revealed a direct relationship between selected environmental factors and symptoms of skin reaction. In particular, the presence of either domestic or courtyard animals is associated with skin pain due to the arthropod bite (OR: 5.25 ; 95\% $\mathrm{Cl}$ : 2.33-28.81; Yates corrected $\chi^{2}=13.68$; $\left.p<0.001\right)$; in addition, the presence of running or stagnant water nearby home location is directly related to skin swelling (OR: 5.69; 95\% Cl: 2.27-14.25; Yates corrected $X^{2}=$ 13.89; $\mathrm{p}<0.001)$

Comparing the frequencies of the risk factors between the settled and the nomad communities, statistically significant differences had been revealed. In particular, in the settled community are more widespread: i) the use of traditional methods (Yates-corrected $x^{2}$ : 12.98; OR: 3.05; 95\% Cl: 1.69- 5.53; p< 0.001); ii) swelling (Yates-corrected $\chi^{2}$ : 36.98; OR: 8.54; 95\% Cl: 4.1217.68 ; $p<0.001$ ); iii) itchiness (Yates-corrected $\chi^{2}$ : 60.55; OR: 18.90; 95\% Cl: 8.14- 43.88; p<0.001). Furthermore, statistical analysis revealed that in the nomad community more frequently recur: i) pain (Yates- corrected $\chi^{2}$ : 18.47; OR: 4.07; 95\% Cl: 2.15- 7.68; $p<$ $0.001)$; ii) presence of running or stagnant water nearby domestic location (Yates-corrected $\mathrm{X}^{2}$ : 60.55; OR: 18.90; 95\% Cl: 8.14- 43.88; $p<0.001)$; iii) presence of domestic/courtyard animals (Yates-corrected $x^{2}$ : 25.79; OR: 4.84; 95\% Cl: 2.63- 8.90; $p<0.001)$. There were no statistically significant differences between the settled and nomad communities in the frequencies of both use of pharmacological products and irritation.

Frequencies of the HHV-8 DNA in mothers' and children's saliva are reported in Table 2.

Overall, we detected HHV-8 DNA in 31 mothers and 15 children. Statistically significant differences in the presence of HHV-8 DNA in mothers' and children's saliva emerged just in the nomad group, whereas in the settled one this difference had not been revealed.

Out of 15 HHV-8 DNA-positive children, 7 (1 male and 6 females) belonged to the nomad community and 8 to the settled one ( 1 male and 7 females). Overall, the sex ratio among children was 13 females vs 2 males $(p=0.03)$ as shown in Table 3.

Moreover, we divided children according to age into two groups: the former included children between 3 and 6 years of age and the latter those between 7 and 13 years. Results indicated that the majority of positive children (11/15) were aged between 7 and 13

Table 2 - HHV-8 DNA in saliva.

\begin{tabular}{|c|c|c|c|c|c|}
\hline Community & $\begin{array}{l}\text { Mothers } \\
\%(n / N) \pm 95 \% \mathrm{Cl}\end{array}$ & $\begin{array}{l}\text { Children } \\
\%(n / N) \pm 95 \% \mathrm{Cl}\end{array}$ & $\begin{array}{l}\mathbf{X}^{2} \\
\text { Yates corrected }\end{array}$ & OR $(95 \% \mathrm{Cl})$ & $p$ \\
\hline Settled & $\begin{array}{l}20.37 \\
(11 / 54) \pm 0.05\end{array}$ & $\begin{array}{l}14.81 \\
(8 / 54) \pm 0.05\end{array}$ & 0.26 & $1.47(0.54-4.00)$ & 0.58 \\
\hline Nomad & $\begin{array}{l}26.32 \\
(20 / 76) \pm 0.05\end{array}$ & $\begin{array}{l}9.2 \\
(7 / 76) \pm 0.05\end{array}$ & 6.49 & $3.52(1.39-8.92)$ & 0.01 \\
\hline Total sample & $\begin{array}{l}23.85 \\
(31 / 130) \pm 0.04\end{array}$ & $\begin{array}{l}\mathbf{1 1 . 5 4} \\
(15 / 130) \pm 0.03\end{array}$ & 5.94 & $2.40(1.17-4.97)$ & 0.01 \\
\hline
\end{tabular}

Cl: confidence interval; OR: odds ratio.

Table 3 - Relationship between HHV-8 DNA in children's saliva and risk factors.

\begin{tabular}{|c|c|c|c|c|c|}
\hline Risk factors & & $\begin{array}{l}\text { HHV-8 DNA in } \\
\text { children's saliva } \\
\%(n / N)\end{array}$ & $\begin{array}{l}\mathbf{X}^{2} \\
\text { Yates corrected }\end{array}$ & $\begin{array}{l}\text { OR } \\
95 \% \mathrm{Cl}\end{array}$ & $\mathbf{p}$ \\
\hline Community & $\begin{array}{l}\text { Settled } \\
\text { Nomadic }\end{array}$ & $\begin{array}{l}46.67(7 / 15) \\
53.33(8 / 15)\end{array}$ & 0.02 & $\begin{array}{l}1.27 \\
0.43-3.73\end{array}$ & 0.20 \\
\hline Gender & $\begin{array}{l}\text { Male } \\
\text { Female }\end{array}$ & $\begin{array}{l}13.33(2 / 15) \\
86.67(13 / 15)\end{array}$ & 5.64 & $\begin{array}{l}4.60 \\
1.90-39.08\end{array}$ & 0.03 \\
\hline Age (years) & $\begin{array}{l}3-6 \\
7-13\end{array}$ & $\begin{array}{l}26.67(4 / 15) \\
73.33(11 / 15)\end{array}$ & 4.71 & $\begin{array}{l}4.25 \\
1.24-13.75\end{array}$ & 0.01 \\
\hline Use of traditional methods\# & $\begin{array}{l}\text { Yes } \\
\text { No }\end{array}$ & $\begin{array}{l}33.33(5 / 15) \\
66.67(10 / 15)\end{array}$ & 0.01 & $\begin{array}{l}0.89 \\
0.28-2.82\end{array}$ & 1.00 \\
\hline
\end{tabular}

\#: use of saliva and/or premasticated herbs; Cl: confidence interval; OR: odds ratio. 
years $(p=0.01)$, included both HHV8 positive male children. There is no statistically significant relationship between the use of traditional methods and the children's positivity to HHV-8 presence in saliva, as reported in Table 3.

\section{Discussion}

In sub-Saharan Africa, several studies proposed that an horizontal mother-to-child HHV-8 transmission through saliva could occur $(11,12,23)$. We suggest that, according to the promoter arthropod hypothesis, the virus could be transmitted through the application of mothers' infected saliva on child's skin (traditional methods) to relieve itching and scratching due to a bloodsucking arthropod bite (19).

Traditional practices as treatment of insect bites are quite widespread in the selected communities: about one third of the interviewed children declared to receive them after a bloodsucking arthropod bite. Furthermore, this habit is significantly more common among mothers from settled community rather than among those from the nomad one, this difference is probably due to the different ways of life of the two populations. The nomad group, in fact, belongs to the Karimojong tribe which exhibits semi-nomadic habits and remained culturally isolated from the rest of Ugandan urban population.

The symptoms of the skin inflammatory reaction after a bloodsucking arthropod bite, in particular skin irritation and swelling, are directly associated with the use of traditional methods. Thus, child's immune response to the bite induces mothers to apply their own saliva at the bite site. In addition, this data underlines the promoting role of the arthropod bite: its blood meal on the young host, which evokes irritation and swelling, increases the children's exposure to maternal saliva and the risk of HHV-8 mother-to-child transmission.

This is one of the first studies which aims to reveal HHV-8 DNA in healthy mothers' and children's saliva living in HHV-8 endemic areas. The prevalence rates of HHV-8 DNA in saliva represent an underestimation of the real seroprevalence because of the intermittent characteristics of the viral salivary shedding. The presence of HHV-8 DNA in not-yet-sexually-active children's saliva confirms that in the selected area intrafamilial horizontal transmission could occur. Moreover, the percentage of positive children increases with age, according to previous seroprevalence studies in this area $(8,11,12)$. However, unexpectedly, we found a significant higher prevalence of the virus in female children's saliva. Despite it is known that African Kaposi Sarcoma affects mainly male population, the infection in endemic areas seems to occur with the same frequency in both gender $(4,6,7,11,12)$. Thus, the data we found are not in agreement with previous studies. One explanation is that female children, who live closer to the mother than their male counterpart, are more exposed to maternal saliva. However, this fact needs to be confirmed by further large-scale studies.

The association between the symptoms of skin inflam- matory reaction and environmental factors indicates that domestic/courtyard animals and water sources could increase the presence of bloodsucking arthropods promoter of HHV-8 infection. In particular, water reservoirs represent micro-environment favourable to life and reproduction of several bloodsucking arthropods which at least for one phase of their lifecycle depend on water (Coquillettidia, Aedes, Ochlerotatus, Culicoides and Leptoconops) (19, 21, 22). Moreover, the presence of domestic/courtyard animals is directly related to skin pain, indicating that this environmental factor could increase the presence of zoophilic bloodsucking arthropods (Phlebotomus and Simulium) (19). These insects are not completely adapted to bite the human host and are able to produce a more intense inflammatory reaction at the bite site.

Comparing data from settled and nomad communities, a statistically significant difference in the use of traditional methods emerges. In line with these data, the use of saliva is more widespread in the settled population than in the nomad one. Moreover, significant difference in the presence of viral DNA in mothers and their children had been recorded just in the nomad community. On the other hand, in the settled group, where a half of children declare to receive their mother's saliva at the bite site, there is no difference in the presence of viral DNA between mothers and respective children.

Thus, in the settled community non-sexual transmission of HHV-8 represents the main source of contagion. In this context the infection is essentially acquired during childhood, whereas in the nomad group the infection seems to occur primarily during adulthood, probably through a sexual route.

HHV-8 DNA was found in both children's and mothers' saliva.

Symptoms of skin reaction due to a bloodsucking arthropod bite and some environmental factors associated with bloodsucking arthropod presence are associated to the use of mother's saliva on children skin to reduce itching and scratching, and thus increase the risk of HHV-8 intrafamilial horizontal transmission. Anyway, further large-scale epidemiological surveys are needed to confirm these findings.

Since we know from literature that saliva of seropositive subjects is the main reservoir of $\mathrm{HHV}-8$, we confirm with this study its presence in both mothers and children. The prove of this alternative, non-sexual horizontal transmission route, could provide the basis for interventions aiming to promote communication companies in order to produce changing behaviour implicated in HHV8 spread, especially among pediatric infection in tropical areas. However, more prospective studies are needing to better define non sexual transmission of HHV8.

\section{Authors' contributions}

RR organised the field survey, analyzed data and edited manuscript. GS performed molecular and statistical analysis, drafted the manuscript. TF performed the database, statistical analysis and drafted the manu- 
script. RG carried out the field survey. VS performed molecular analyses. MF organised and supervised the field survey. PGM performed statistical analyses and drafted the manuscript. GA supervised the molecular analyses and edited the manuscript. CM conceived the study and edited the manuscript. All authors read and approved the final manuscript.

\section{Acknowledgements}

We are particularly grateful to all the children interviewed, the technical staff for their support, the schools and health care service directors for their collaborative attitude throughout the investigation.

\section{Competing interests}

The authors declare that they have no competing interests.

\section{References}

1. Chang $\mathrm{Y}$, Cesarman E, Pessin MS, et al. Identification of herpesvirus-like DNA sequences in AIDS-associated Kaposi's sarcoma. Science. 1994; 266:1865-1869.

2. Soulier J, Grollet L, Oksenhendler E, et al. Kaposi's sarcoma-associated herpesvirus-like DNA sequences in multicentric Castleman's disease. Blood. 1995; 86:1276-1280.

3. Cesarman E, Chang Y, Moore PS, et al. Kaposi's sarcomaassociated herpesvirus-like DNA sequences in AIDS-related body-cavity-based lymphomas. N Engl J Med. 1995; 332:1186-1191.

4. Parkin DM, Sitas F, Chirenje M, et al. Part I: Cancer in Indigenous Africans-burden, distribution and trends. The Lancet Oncol. 2008; 9:682-692.

5. Pinheiro Rdos S, Ferreira Dde C, Nóbrega F, et al. Current status of herpesvirus identification in the oral cavity of HIVinfected children. Rev Soc Bras Med Trop. 2013; 46(1):1519.

6. Dedicoat M, Newton R. Review of the distribution of Kaposi' s sarcoma associated herpesvirus (KSHV) in Africa in relation to the incidence of Kaposi' s sarcoma. $\mathrm{Br} \mathrm{J}$ Cancer. 2003; 88:1-3.

7. Dollard SC, Butler LM, Jones AM, et al. Substantial regional differences in human herpesvirus 8 seroprevalence in sub-Saharan Africa: insights on the origin of the "Kaposi's sarcoma belt". Int J Cancer. 2010; 127:2395-2401.

8. Serraino D, Toma L, Buttò $S$, et al. A seroprevalence study of humanherpesvirus type 8 (HHV8) in eastern and Central Africa and in the Mediterranean area. Eur J Epidemiol. $2001 ; 17: 871-876$.
9. De Souza VA, Sumita LM, Nascimento MC, et al. Human Herpes virus- 8 infection and oral shedding in Amerindian and non Amerindian populations in the Brazilian Amazon region. J Infect Dis. 2007; 96:844-852.

10. Cunha AM, Caterino-de-Araujo A, Costa SC, et al. Increasing seroprevalence of human Herpesvirus $8(\mathrm{HHV}-8)$ with age confirms HHV-8 endemicity in Amazon Amerindians from Brazil. J Gen Virol. 2005; 9:2433-2437.

11. Butler LM, Were WA, Downing R, et al. Human Herpesvirus 8 infection in children and adults in a populationbased study in rural Uganda. J Infect Dis. 2011; 203:625634.

12. Mbulaiteye SM, Pfeiffer RM, Whitby D, et al. Human herpesvirus 8 infection within families in rural Tanzania. J Infect Dis. 2003; 187:1780-1785.

13. De Franca TR, De Araùjo RA, Ribeiro CM, Leao JC. Salivary shedding of HHV-8 in people infected or not by human immunodeficiency virus 1. J Oral Pathol Med. 2011; 40:97-102.

14. Taylor MM, Chohan B, Lavreys L, et al. Shedding of human herpesvirus 8 in oral and genital secretions from HIV1 -seropositive and -seronegative Kenyan women. J Infect Dis. 2004; 190:484-488.

15. Perna AM, Bonura F, Vitale F, et al. Antibodies to human Herpesvirus 8 (HHV8) in general population and in individuals at risk for sexually transmitted diseases in Western Sicily. Int J Epidemiol. 2000; 1:175-179.

16. Butler LM, Neilands TB, Mosam Mzolo S, Martin JN. A population-based study of how children are exposed to saliva in KwaZulu-Natal Province, South Africa: implications for the spread of saliva-borne pathogens to children. Trop Med Int Health. 2010; 15:442-453.

17. Wakeham K, Webb EL, Sebina I, et al. Risk factors for seropositivity to Kaposi's sarcoma associated herpesvirus (KSHV) among children in Uganda. J Acquir Immune Defic Syndr. 2013; 63:228-233.

18. Wojcicki JM. Traditional behavioural practices, the exchange of saliva and HHV-8 transmission in sub-Saharan African populations. Br J Cancer. 2003; 89:2016-2017.

19. Coluzzi M, Manno D, Guzzinati S, et al. The bloodsucking arthropod bite as possible cofactor in the transmission of human herpesvirus-8 infection and in the expression of Kaposi's sarcoma disease. Parassitol. 2002; 44:122-123.

20. Andrade BB, Teixeira CR, Barral A, Barral-Netto M. Haematophagous arthropod saliva and host defense system: a tale of tear and blood. Ann Braz Ac Sciences. 2005; 77:665-693.

21. Ascoli V, Senis G, Zucchetto A, et al. Distribution of "promoter" sandflies associated with incidence of classic Kaposi's sarcoma. Med Vet Entomol. 2009; 3:217-225.

22. Ascoli V, Facchinelli L, Valerio L, et al. Kaposi's sarcoma, human herpesvirus 8 infection and the potential role of promoter-arthropod bites in northern Sweden. J Med Virol. 2006; 11:1452-1455.

23. Ronher E, Wyss N, Trelle S, et al. HHV8 seroprevalence: a global view. Sistematic Reviews. 2014; 3:11. 